\begin{tabular}{|c|c|}
\hline Title & $\begin{array}{l}\text { A daptive Subdomain Model Order Reduction With Discrete Empirical Interpolation Method for Nonlinear Magneto- } \\
\text { Quasi-Static Problems }\end{array}$ \\
\hline Author(s) & Sato, Y uki; Clemens, Markus; Igarashi, Hajime \\
\hline Citation & $\begin{array}{l}\text { IEEE transactions on magnetics, 52(3), } 1100204 \\
\text { https://doi.org/10.1109/T MA G.2015.2489264 }\end{array}$ \\
\hline Issue Date & 2016-03 \\
\hline Doc URL & http://hdl .handle.net/2115/61517 \\
\hline Rights & $\begin{array}{l}\text { C } 2015 \text { IEEE. Personal use of this material is permitted. Permission from IEEE must be obtained for all other uses, in } \\
\text { any current or future media, including reprinting/republishing this material for advertising or promotional purposes, } \\
\text { creating new collective works, for resale or redistribution to servers or lists, or reuse of any copyrighted component of } \\
\text { this work in other works. }\end{array}$ \\
\hline Type & article (author version) \\
\hline File Information & A daptive Subdomain_full.pdf \\
\hline
\end{tabular}

Instructions for use 


\title{
Adaptive Subdomain Model Order Reduction with Discrete Empirical Interpolation Method for Nonlinear Magneto-Quasi-Static Problems
}

\author{
Yuki Sato ${ }^{1,2}$, Markus Clemens ${ }^{3}$, Senior Member IEEE, Hajime Igarashi ${ }^{1}$, Member IEEE \\ ${ }^{1}$ Graduate School of Information Science and Technology, Hokkaido University, 060-0814 Sapporo, Japan \\ ${ }^{2}$ Research Fellow of Japan Society for the Promotion of Science (JSPS), Tokyo, 102-0083, Japan \\ ${ }^{3}$ Bergische Universität Wuppertal, FB E, Chair of Electromagnetic Theory, Wuppertal, D-42119, Germany
}

\begin{abstract}
This paper presents a novel adaptive subdomain model order reduction (MOR) based on proper orthogonal decomposition (POD) and discrete empirical interpolation (DEI) methods for nonlinear magneto-quasi-static (MQS) problems. In this method, a nonlinear region is decomposed into two regions where one of the regions includes all those finite elements which have particularly strong saturation and the other region does not. MOR based on POD and DEI methods is applied only to the latter region. Both regions are determined automatically at each time step. It is shown that this method can effectively reduce the computational time to solve the nonlinear MQS problems without losing quality of accuracy.
\end{abstract}

Index Terms - Finite element method, magneto-quasi-static field, model order reduction, nonlinear problem.

\section{INTRODUCTION}

$\mathrm{M}$ ODEL ORDER REDUCTION (MOR) has been applied to a variety of discrete electromagnetic field problems, for example, the transient electro- and magneto-quasi-static (EQS and MQS) problems [1]-[8]. Although we can apply MOR based on proper orthogonal decomposition (POD) to nonlinear problems, this method may not be remarkably effective for analyzing the nonlinear problems [1][2]. There are two problems in POD-based MOR: one is a deterioration of the accuracy in saturated regions and another is an increase of computational cost in iterative processes in which a nonlinear term has to be updated and matrix-matrix products have to be calculated at each time step.

To tackle the deterioration of the accuracy, one of the authors has proposed subdomain MOR [3][4] in which the whole region is decomposed into linear and nonlinear regions. Then MOR is applied only to the linear region. This approach helps to maintain the accuracy in the saturated region compared with the non-reduced model. This method has been successfully applied to the optimization problems for magnetostatic [5] and high-frequency problems [6]. However, this method cannot dramatically reduce the computational cost because the number of degrees of freedom (DoF) is much larger than that of the usual approach where MOR is applied to the whole computational domain.

On the other hand, MOR based on discrete empirical interpolation (DEI) method [7]-[10] has been proposed to

Manuscript received June 20, 2015. Corresponding author: Y. Sato (email: yukisato@em.ist.hokudai.ac.jp).

Digital Object Identifier (inserted by IEEE). reduce the computational cost in the iterative processes. In this method, the nonlinear term is interpolated in the localized nonlinear regions which are determined by evaluating nonlinear terms obtained during a preconditioning process.

In this paper, we propose a novel POD-based MOR method combining subdomain MOR and DEI methods in which a nonlinear region is decomposed into two regions. One has finite elements which exhibit a particularly strong nonlinear behavior in the ferromagnetic material while the other does not have them. POD-based MOR is applied only to the latter region. These regions should be determined automatically because the electromagnetic field distributions in the nonlinear ferromagnetic material change at each time step and it is initially not known where the elements which exhibit strong nonlinearity due to ferromagnetic saturation effects are positioned before analyzing the electromagnetic field distributions. Therefore, these regions are automatically determined from the field at the previous time step. Moreover, the DEI method is applied to the nonlinear term in the latter region to reduce the computational time in the iterative processes.

In this work, we apply the present method to the inductor and reactor models for MQS problems and compare the present method with the conventional MOR, DEI-based MOR and subdomain MOR with respect to the accuracy and the computational time.

\section{Magneto-Quasi-Static Analysis}

We employ finite element method (FEM) to solve an MQS system in which the governing equations derived from Maxwell equations are expressed as:

$$
\operatorname{rot} v(\boldsymbol{A}) \operatorname{rot} \boldsymbol{A}+\sigma\left(\frac{\partial \boldsymbol{A}}{\partial t}+\operatorname{grad} \frac{\partial \varphi}{\partial t}\right)=\boldsymbol{J}
$$


$\operatorname{div} \sigma\left(\frac{\partial \boldsymbol{A}}{\partial t}+\operatorname{grad} \frac{\partial \varphi}{\partial t}\right)=0$

where $\boldsymbol{A}, \varphi, \boldsymbol{J}, v$ and $\sigma$ are vector and scalar potentials, current density, magnetic reluctivity and conductivity, respectively. We apply the weighted residual method to (1) to obtain

$$
\begin{aligned}
& \sum_{i} a_{i} \int_{\Omega} \operatorname{rot} \boldsymbol{N}_{j} \cdot V(\boldsymbol{A}) \operatorname{rot} \boldsymbol{N}_{i} \mathrm{~d} V+\sum_{i} \frac{\mathrm{d} a_{i}}{\mathrm{~d} t} \int_{\Omega} \sigma \boldsymbol{N}_{i} \cdot \boldsymbol{N}_{j} \mathrm{~d} V \\
& +\sum_{k} \frac{\mathrm{d} \varphi_{k}}{\mathrm{~d} t} \int_{\Omega} \sigma \boldsymbol{N}_{j} \cdot \operatorname{grad} w_{k} \mathrm{~d} V=\int_{\Omega} \boldsymbol{N}_{j} \cdot \boldsymbol{J} \mathrm{d} V \\
& \sum_{i} \frac{\mathrm{d} a_{i}}{\mathrm{~d} t} \int_{\Omega} \sigma \boldsymbol{N}_{i} \cdot \operatorname{grad} w_{u} \mathrm{~d} V \\
& \quad+\sum_{k} \frac{\mathrm{d} \varphi_{k}}{\mathrm{~d} t} \int_{\Omega} \sigma \operatorname{grad} w_{k} \cdot \operatorname{grad} w_{u} \mathrm{~d} V=0
\end{aligned}
$$

where $\boldsymbol{N}_{i}$ and $w_{j}$ are the vector and scalar interpolation functions, respectively. Moreover, if the electromagnetic device is connected to the electric circuit as shown in Fig. 1, we consider the electric circuit equation

$V=R i+\frac{\mathrm{d} \Phi}{\mathrm{d} t}$

where $\Phi$ is the magnetic flux across a coil. We solve (2) and (3) using the incomplete Cholesky conjugate gradient method.

\section{ADAPTIVE SUBDOMAIN MOR WITH DEI METHOD}

\section{A. Formulation}

We rewrite (2) and (3) to $A \boldsymbol{x}+\boldsymbol{b}+\boldsymbol{f}(\boldsymbol{x})=0$ in which the first and the second terms are linear while the third term is nonlinear. In this method, the unknown $\boldsymbol{x}$ is decomposed into three parts: $\boldsymbol{x}_{\text {strong }}, \boldsymbol{x}_{\text {weak }}$ and $\boldsymbol{x}_{\text {linear }}$. The vectors $\boldsymbol{x}_{\text {strong }}$ and $\boldsymbol{x}_{\text {weak }}$ compose the unknowns for the finite elements which have the strong and weak nonlinearities while $\boldsymbol{x}_{\text {linear }}$ is the unknown for the finite elements in a linear domain. From this subdivision, we can obtain

$$
\left[\begin{array}{lll}
\mathrm{A}_{11} & \mathrm{~A}_{12} & \mathrm{~A}_{13} \\
\mathrm{~A}_{21} & \mathrm{~A}_{22} & \mathrm{~A}_{23} \\
\mathrm{~A}_{31} & \mathrm{~A}_{32} & \mathrm{~A}_{33}
\end{array}\right]\left[\begin{array}{c}
\boldsymbol{x}_{\text {strong }} \\
\boldsymbol{x}_{\text {weak }} \\
\boldsymbol{x}_{\text {linear }}
\end{array}\right]+\left[\begin{array}{c}
\boldsymbol{b}_{\text {strong }} \\
\boldsymbol{b}_{\text {weak }} \\
\boldsymbol{b}_{\text {linear }}
\end{array}\right]+\left[\begin{array}{c}
\boldsymbol{f}_{\text {strong }}(\boldsymbol{x}) \\
\boldsymbol{f}_{\text {weak }}(\boldsymbol{x}) \\
0
\end{array}\right]=0
$$

where $\mathrm{A}_{11} \in \mathrm{R}^{p \times p}, \mathrm{~A}_{12} \in \mathrm{R}^{p \times q}, \mathrm{~A}_{13} \in \mathrm{R}^{p \times(n-l)}, \mathrm{A}_{21} \in \mathrm{R}^{q \times p}, \mathrm{~A}_{22} \in \mathrm{R}^{q \times q}$, $\mathrm{A}_{23} \in \mathrm{R}^{q \times(n-l)}, \quad \mathrm{A}_{31} \in \mathbb{R}^{(n-l) \times p}, \quad \mathrm{~A}_{32} \in \mathrm{R}^{(n-l) \times q}, \quad \mathrm{~A}_{33} \in \mathrm{R}^{(n-l) \times(n-l)}$, $\boldsymbol{x}_{\text {strong }}, \boldsymbol{b}_{\text {strong }} \in \mathbb{R}^{p}, \boldsymbol{x}_{\text {weak }}, \boldsymbol{b}_{\text {weak }} \in \mathbb{R}^{q}, \boldsymbol{x}_{\text {linear }}, \boldsymbol{b}_{\text {linear }} \in \mathbb{R}^{(n-l)}, p$ and $q$ $(l=p+q)$ are the number of DoFs in the domains which have strong and weak nonlinearities. In this method, we apply POD-based MOR to $\boldsymbol{x}_{\text {weak }}$ and $\boldsymbol{x}_{\text {linear. The data matrices are }}$ constructed in the preconditioning process

$$
\mathrm{X}_{\text {weak }}=\left[\begin{array}{llll}
\boldsymbol{x}_{\text {weak }}\left(t_{1}\right) & \boldsymbol{x}_{\text {weak }}\left(t_{2}\right) & \cdots & \boldsymbol{x}_{\text {weak }}\left(t_{s}\right)
\end{array}\right]
$$

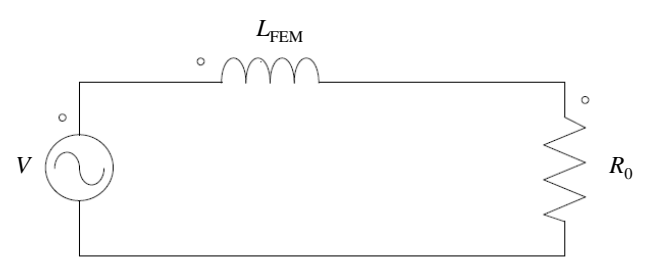

Fig. 1 Circuit connected to FE model

$X_{\text {linear }}=\left[\begin{array}{llll}x_{\text {linear }}\left(t_{1}\right) & \boldsymbol{x}_{\text {linear }}\left(t_{2}\right) & \cdots & \boldsymbol{x}_{\text {linear }}\left(t_{s}\right)\end{array}\right]$

where $s$ is number of snapshots. The singular value decomposition applied to matrices in (5) leads to

$\mathrm{X}_{\text {weak }}=\mathrm{W}_{\text {weak }} \Sigma_{\text {weak }} \mathrm{V}_{\text {weak }}^{t}, \mathrm{X}_{\text {linear }}=\mathrm{W}_{\text {linear }} \Sigma_{\text {linear }} \mathrm{V}_{\text {linear }}^{t}$

where $\mathrm{W}_{\alpha}$ and $\mathrm{V}_{\alpha}, \alpha=\{$ weak, liner $\}$, represent the matrices whose columns are the eigenvectors of $\mathrm{X}_{\alpha} \mathrm{X}_{\alpha}{ }^{t}$ and $\mathrm{X}_{\alpha}{ }^{t} \mathrm{X}_{\alpha}$. We construct the transformation matrix to reduce the DoF in (4)

$\boldsymbol{x}=\mathrm{W} \boldsymbol{y}=\left[\begin{array}{ccc}\mathrm{I} & 0 & 0 \\ 0 & \mathrm{~W}_{\text {weak }} & 0 \\ 0 & 0 & \mathrm{~W}_{\text {linear }}\end{array}\right] \boldsymbol{y}$

where $y \in \mathrm{R}^{p+s+s}$. Substituting (7) into (4), we obtain the reduced equation

$\mathrm{W}^{t} \mathrm{AW} \boldsymbol{y}+\mathrm{W}^{t} \boldsymbol{b}+\mathrm{W}^{t} \boldsymbol{f}(\mathrm{W} \boldsymbol{y})=0$

Solution of (8) needs long computational time because we have to update the nonlinear term and perform matrix-matrix product in the Newton Raphson iterations. To circumvent this difficulty, we apply DEI method to $\boldsymbol{f}_{\text {weak }}(\boldsymbol{x})$. Namely, $\boldsymbol{f}_{\text {weak }}$ is approximated as

$\boldsymbol{f}_{\text {weak }}(\boldsymbol{x}) \approx \mathrm{U}\left(\mathrm{P}^{t} \mathrm{U}\right)^{-1} \mathrm{P}^{t} f_{\text {weak }}(\boldsymbol{x})$

where $\mathrm{U}$ and $\mathrm{P}$ can be found in [7]. From this approximation of the nonlinear term, we can rewrite (8) as

$\mathrm{W}^{t} \mathrm{AW} \boldsymbol{y}+\mathrm{W}^{t} \boldsymbol{b}+\mathrm{W}^{t} \mathrm{~W}_{\mathrm{DEI}} \boldsymbol{f}(\mathrm{W} \boldsymbol{y})=0$

where

$\mathrm{W}_{\mathrm{DEI}}=\left[\begin{array}{ccc}\mathrm{I} & 0 & 0 \\ 0 & \mathrm{U}\left(\mathrm{P}^{t} \mathrm{U}\right)^{-1} \mathrm{P}^{t} & 0 \\ 0 & 0 & \mathrm{I}\end{array}\right]$

The linearized equation derived from (10)

$\frac{\partial \boldsymbol{g}(\mathrm{W} y)}{\partial \boldsymbol{y}} \delta \boldsymbol{y}=-\boldsymbol{g}$ 
is solved iteratively where $\boldsymbol{g}$ denotes the residual in (10). The Jacobi matrix in (12) is decomposed to linear and nonlinear matrices

$$
\frac{\partial \boldsymbol{g}(\mathrm{W} \boldsymbol{y})}{\partial \boldsymbol{y}}=\mathrm{W}^{t} \mathrm{AW}+\mathrm{W}^{t} \mathrm{~W}_{\mathrm{DEI}} \frac{\partial \boldsymbol{f}(\mathrm{W} \boldsymbol{y})}{\partial \boldsymbol{x}} \mathrm{W}
$$

The second term of (13) has to be updated. The computational cost for this process is much smaller than that of (8) because of the structure of $\mathrm{W}_{\mathrm{DEI}}$. The coefficient matrix of (12) is, however, non-symmetric. We can employ a linear solver for non-symmetric matrices such as the biconjugate gradient (BiCG), generalized minimal residual method. In this work, we employ BiCG method.

\section{B. Adaptive subdomain determination}

In this method, we decompose the nonlinear region into two regions. The computational time and accuracy of this method depend on the way of subdivision. For domain subdivision, we pay attention to the $\mathrm{BH}$ characteristics. That is, we a priori subdivide the domain of B into weakly and strongly nonlinear ones on the basis on the BH curve as shown in Fig. 2.

The electromagnetic field distributions in the nonlinear ferromagnetic material change at each time step. Moreover, it is initially not known where the elements which exhibit strong nonlinearity due to ferromagnetic saturation effects are positioned before analyzing the electromagnetic field distributions. From these reasons, we propose the adaptive domain decomposition whose algorithm is described below :

--Adaptive subdomain determination method----

\section{in preconditioning process}

for $n=0, \ldots, s$

1. Solve $\mathrm{A} \boldsymbol{x}_{n}+\boldsymbol{b}+\boldsymbol{f}\left(\boldsymbol{x}_{n}\right)=0$ at time step $n$.

2. Decomopse the nonlinear region into two regions based on B-H curve.

3. Construct the matrices $\mathrm{W}$ and $\mathrm{W}_{\mathrm{DEI}}$ for time step $n$.

end for

\section{in iterative process}

for $n=s+1, \ldots, N$

4. Solve $\mathrm{W}^{t} \mathrm{AW} \boldsymbol{y}_{n}+\mathrm{W}^{t} \boldsymbol{b}+\mathrm{W}^{t} \mathrm{~W}_{\mathrm{DE}} \boldsymbol{f}\left(\mathrm{W} \boldsymbol{y}_{n}\right)=0$ at time step $n$.

5. Detect the solution which does not significantly change from the solutions obtained in preconditioning process.

6. Choose $\mathrm{W}$ and $\mathrm{W}_{\mathrm{DEI}}$ based on the detection results in process 5 .

end for

The above mentioned algorithm is illustrated in Fig.3.

\section{NUMERICAL RESUltS}

To test the performance of the present method, we apply usual MOR, DEI-based MOR, subdomain MOR and the present method to the inductor and reactor shown in Figs. 4 and 5 which are connected to the simple circuit shown in Fig. 1 where $R_{0}$ is set to $10^{-5} \Omega$ and $V$ in the inductor and reactor models is $0.008 \mathrm{~V}$ and $0.015 \mathrm{~V}$, respectively. The driving frequency is $100 \mathrm{~Hz}$ and $\Delta t$ is $2.5 \times 10^{-4}$ seconds. The core

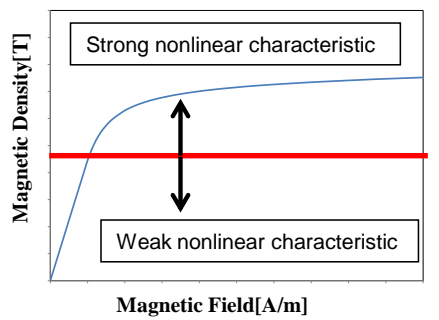

Fig.2 BH curve

- Solve $A \boldsymbol{x}+\boldsymbol{b}+\boldsymbol{f}(\boldsymbol{x})=0$
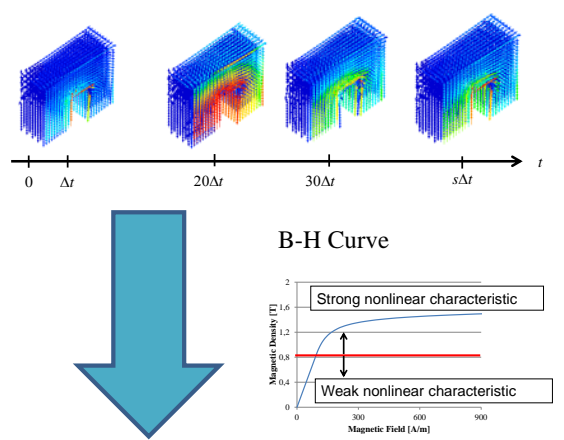

- Subdivide the nonlinear region
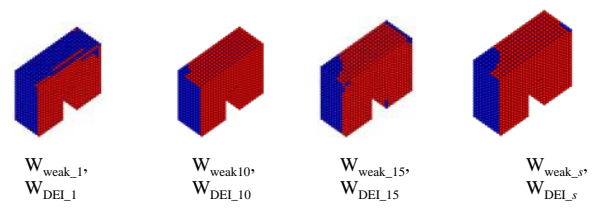

(a)Preconditioning process

- Solve $\mathrm{W}^{t} \mathrm{AW} \boldsymbol{y}+\mathrm{W}^{t} \boldsymbol{b}+\mathrm{W}^{t} \mathrm{~W}_{\mathrm{DE}} \boldsymbol{f}(\boldsymbol{x})=0$
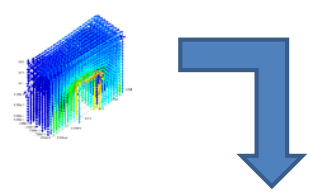

Compare to Solutions obtained in preconditioning process.

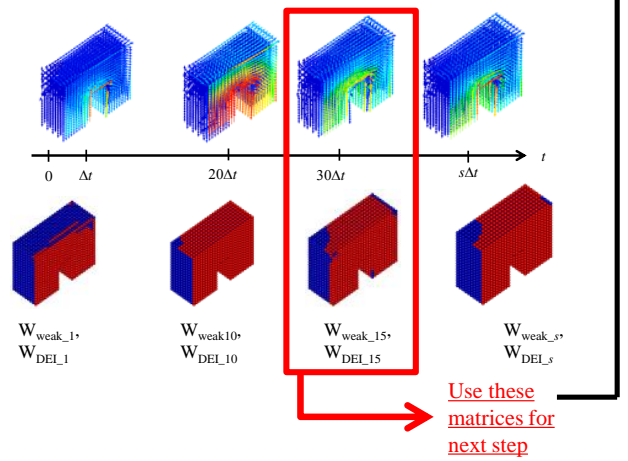

(b)Iterative process

Fig. 3 Algorithm for adantive subdomain determination method. material is assumed to be 50A400. The initial states are assumed to be zero fields. The number of snapshots is set to $s=50$ in both models. Due to symmetry, we analyze one eighth of the domain in both models.

To compare the present method with the other MOR methods, the error is defined by

Error $=\frac{1}{N_{t}} \sum_{n=1}^{N_{t}} \sqrt{\frac{\sum_{k=1}^{N_{e}}\left|\boldsymbol{B}_{k, n}-\boldsymbol{B}_{k, n}^{\mathrm{MOR}}\right|^{2}}{\sum_{k=1}^{N_{e}}\left|\boldsymbol{B}_{k, n}\right|^{2}}}$ 
where $N_{t}, N_{e}, \boldsymbol{B}_{k, n}$ and $\boldsymbol{B}_{k, n}{ }^{\mathrm{MOR}}$ are the total time steps, the number of elements, the magnetic induction in $k$-th element at time step $n$ obtained by FEM and MOR methods.

To compare the computational time, we define the speedup ratio $\tau_{\mathrm{FEM}} / \tau_{\mathrm{MOR}}$ where $\tau_{\mathrm{FEM}}$ and $\tau_{\mathrm{MOR}}$ are the computational times for conventional FEM and MOR methods, respectively. The computational time does not include the tasks for the preconditioning process of MOR in which we snapshot the electromagnetic fields and construct transformation matrix.

The results of the speedup ratio and error are shown in TABLE I and II. The DEI-based MOR performs best and worst with respect to the speedup ratio and the error, respectively, while the subdomain MOR behaves just the opposite in both models. These methods have trade-off property between the error and the speedup ratio. The MOR and subdomain MOR cannot shorten the computational time. The reason for these results is that the Newton-Raphson iteration takes long time for convergence so that large number of matrix-matrix products is required. Note that DEI can reduce the computational time for matrix-matrix products. We can see in TABLE I and II that the errors of the present method are almost the same as those of subdomain MOR while the speedup ratios of the present method are more than 3.5 in both models. The temporal changes in errors are plotted in Fig. 6 in which the maximum errors for subdomain and present method are less than $1 \%$ while those of the others are about $10 \%$. It is concluded from these results that the presented method is superior over other MOR approaches in the sense that the latter cannot be simultaneously more accurate and faster than the former.

\section{CONCLUSION}

In this paper, we have proposed an adaptive subdomain MOR with DEI method for nonlinear MQS problems. We have compared the performance of FEM, usual MOR, DEIbased MOR, subdomain MOR with that of present method. It has been concluded that the present method outperforms other MOR approaches with respect to accuracy and computational speed.

\section{ACKNOWLEDGMENT}

This work was supported in part by JSPS KAKENHI Grant Number 25630101 and 15J01550.

\section{REFERENCES}

[1] D. Schmidthäusler and M. Clemens, "Low-Order Electroquasistatic Field Simulations Based on Proper Orthogonal Decomposition," IEEE Trans. Magn., vol. 48, no. 2, pp. 567-570, Feb. 2012.

[2] Y. Sato and H. Igarashi, "Model Reduction of Three-Dimensional Eddy Current Problems Based on the Method of Snapshots," IEEE Trans. Magn, vol. 49, no. 5, pp. 1697-1700, May, 2013.

[3] D. Schmidthäusler S. Schöps and M. Clemens, "Reduction of Linear Subdomains for Non-Linear Electro-Quasistatic Field Simulation," IEEE Trans. Magn., vol. 49, no. 5, Feb., 2013.

[4] D. Schmidthäusler S. Schöps and M. Clemens, "Linear Subspace Reduction for Quasistatic Field Simulations to Accelerate Repeated Computations," IEEE Trans. Magn., vol. 50, no. 2, Feb., 2014.
TABLE I

SPEEDUP RATIOS OF MORS.

\begin{tabular}{|c|c|c|c|c|}
\hline & MOR & SubMOR & DEI & Present \\
\hline Inductor & 0.687 & 0.850 & 12.3 & 3.61 \\
\hline Reactor & 1.19 & 0.992 & 24.9 & 5.48 \\
\hline \multicolumn{5}{|c|}{$\begin{array}{c}\text { TABLE II } \\
\text { ERROR IN MORS. }\end{array}$} \\
\hline & MOR & SubMOR & DEI & Present \\
\hline Inductor & $3.45 \%$ & $0.749 \%$ & $5.47 \%$ & $0.982 \%$ \\
\hline Reactor & $1.87 \%$ & $0.245 \%$ & $2.65 \%$ & $0.213 \%$ \\
\hline
\end{tabular}

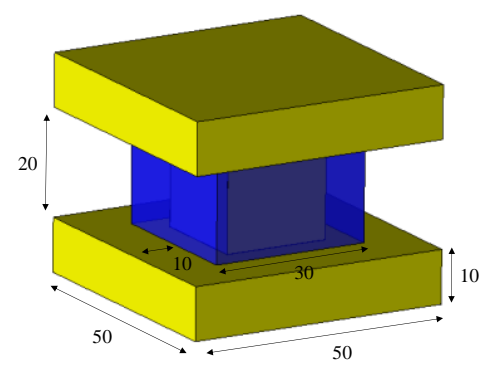

Fig. 4 Inductor model.(yellow : core, blue : coil, size [mm]).

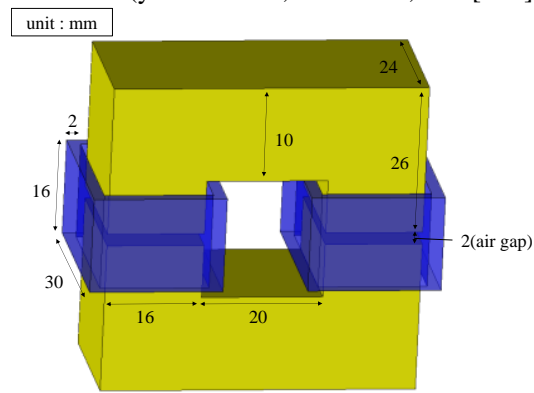

Fig. 5 Reactor model.(yellow : core, blue : coil, size [mm])

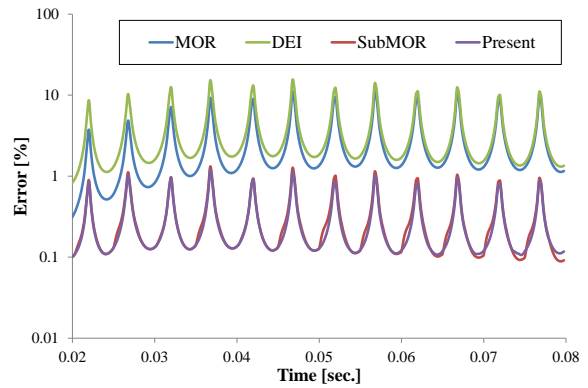

Fig. 6 Error with respect to time for each MOR in reactor model..

[5] Y. Sato, H. Igarashi, "Model Order Reduction Applied to Optimization of Electromagnetic Devices," Proceeding of ISTET2013, Pilsen, Czech Republic, June, 2013.

[6] Y. Sato, F. Campelo and H. Igarashi, "Fast Shape Optimization of Antennas Using Model Order Reduction," IEEE Trans. Magn. vol. 51, no. 3, Mar. 2015.

[7] T. Henneron and S. Clénet, "Model Order Reduction of Nonlinear Magnetostatic Problems Based on POD and DEI Methods," IEEE Trans. Magn, vol. 50, no. 2, Feb., 2014.

[8] T. Henneron and S. Clénet, "Model-Order Reduction of Multi-Input Non-Linear Systems Based on POD and DEI Methods," IEEE Trans. Magn, vol. 51, no. 3, Mar.., 2015.

[9] S. Chaturantabut and D. C. Sorensen, "Nonlinear Model Reduction via Discrete Empirical Interpolation," SIAM J. Sci. Comput., vol. 32, no. 5, pp. 2737-2764, 2010.

[10] B. Preherdtorfer, D. Butnaru, K. Willcox and H. Bungartz, "Localized Discrete Empirical Interpolation Method," SIAM J. Sci. Comput., vol. 36, no. 1, pp. A168-A192, 2014. 